Masayo Nomura $\cdot$ Yoh-ichiro Hamasaki

Ichiro Katayama · Kuniko Abe · Norio Niikawa

Koh-ichiro Yoshiura

\title{
Eosinophil infiltration in three patients with generalized atrophic benign epidermolysis bullosa from a Japanese family: molecular genetic and immunohistochemical studies
}

Received: 19 May 2005 / Accepted: 18 July 2005/Published online: 20 September 2005

(C) The Japan Society of Human Genetics and Springer-Verlag 2005

\begin{abstract}
Generalized atrophic benign epidermolysis bullosa (GABEB), a subtype of epidermolysis bullosa (EB), is an autosomal recessive skin disease characterized by derm-epidermal separation leading to skin fragility and atrophy and other associated abnormalities. Although a few reports demonstrated that eosinophils are infiltrating beneath bullas in infants with some types of EB, no such condition in adult GABEB patients has been known. Here we report on three adult patients with GABEB from a Japanese family, whose bullous skin lesions showed massive eosinophil infiltration. One of the three patients showed amyloid deposition at the intestine, kidney, and skin. Linkage analysis revealed that GABEB in the family was linked to COL17 Al with a maximum LOD score of 3.08. Mutation analysis identified in the three patients a homozygous insertional mutation, 209-210insCA, in exon 5 of COL17A1. The expression of mutated COL17AI was confirmed by
\end{abstract}

\footnotetext{
M. Nomura $\cdot$ N. Niikawa $\cdot$ K. Yoshiura $(\bowtie)$

Department of Human Genetics, Nagasaki University Graduate School of Biomedical Sciences, 1-12-4 Sakamoto,

Nagasaki 852-8523, Japan

E-mail: kyoshi@net.nagasaki-u.ac.jp

Tel: $+81-956-8497120$

Fax: + 81-956-8497121

Y. Hamasaki

Department of Dermatology,

Dokkyo University School of Medicine,

Tochigi, Japan

I. Katayama

Department of Dermatology,

Osaka University Graduate School of Medicine,

Suita, Japan

K. Abe

Department of Pathology,

Nagasaki University Hospital,

Nagasaki, Japan

N. Niikawa $\cdot$ K. Yoshiura

CREST, Japan Science and Technology Agency (JST),

Kawaguchi, Japan
}

semiquantitative RT-PCR, but no signals for truncated COL17A1 protein were detected by the immunohistochemical studies using antibodies against BP180. Furthermore, no autoantibody against the mutant protein was detected by western blot analysis. It is thus less likely that autoantibody and/or a local immune reaction in their skin has a primary role in eosinophil infiltration in these patients.

Keywords Generalized atrophic benign epidermolysis bullosa (GABEB) - Bullous pemphigoid - BP180 . COL17A1 $\cdot$ Eosinophil infiltration · Amyloidosis

\section{Introduction}

Generalized atrophic benign epidermolysis bullosa (GABEB, OMIM \#226650), a subtype of epidermolysis bullosa (EB), is an autosomal recessive disease and characterized by blistering derm-epidermal separation leading to skin fragility and atrophy, nail dystrophy, dental anomalies, and alopecia of the scalp, eyebrows, secondary sexual hair (Hinter and Wolf 1982). GABEB is caused by mutations in several genes, including those for collagen type XVII alpha-1 (COL17A1 mapped at 10q24.3), laminin gamma-2 (LAMC2 at 1q25-31), laminin beta-3 ( $L A M B 3$ at 1q32), and laminin alpha-3 ( $L A M A 3$ at $18 \mathrm{q} 11.2)$. A number of truncation and missense mutations in COL17A1 have been reported (Schumann et al. 1997; Bauer and Lanschuetzer 2003). The skin fragility of GABEB may be due to lack of the COL17A1 gene product (collagen XVII, also called BPAG2 or BP180).

Bullous pemphigoid (BP) is an acquired autoimmune skin disease characterized by multitense blisters due to derm-epidermal detachment and by prominent involvement of eosinophils around blisters (Van den Bergh and Giudice 2003). It is known that anti-BP180 autoantibody, which recognizes the extracellular domain of BP180 (Nc16A), gives rise to an antibody-mediated 
inflammatory reaction in the basement membrane zone (BMZ) and derm-epidermal separation leading to blister formation in BP patients. Eosinophils around the blisters are activated at $\mathrm{BMZ}$ before and simultaneously with the separation. Although eosinophils may play an important role in damaging $\mathrm{BMZ}$ and accelerating the blister formation (Van den Bergh and Giudice 2003), the underlying mechanism to produce an autoantibody against COL17A1 and induce eosinophil infiltration around the blisters is not yet understood.

In contrast, an association of EB with eosinophil/ mast cell infiltrations in the bulla is rare and, to our knowledge, such an association has been reported only in a few infants with some types of EB other than GABEB (Sanz-Santamaria and McNutt 1966; AntonLamprecht and Schnyder 1982; Roth et al. 1990; Grunwald et al. 1999); however, the cause of eosinophil infiltration remains unknown. Furthermore, in a few patients with autosomal recessive dystrophic EB (OMIM \#226600) due to mutations in (COL7A1), amyloidosis was seen to cause renal dysfunction (Fine et al. 2004). However, there is no report of GABEB patients with amyloidosis.

We recently encountered three patients with GABEB who were the children of two consanguineous couples in a Japanese family. In addition to clinical manifestations for GABEB, the patients had distinct histological features characterized by massive eosinophil infiltration around blisters. One of three patients also had massive depositions of amyloid in the intestinal epithelium and kidney and, to a lesser degree, in the skin. Here we show the results of genetic and immunohistochemical studies of this family.

\section{Materials and methods}

\section{Patients and their clinical findings}

The proband (IV-3, Fig. 1), a 56-year-old female, is the second child of healthy parents who were cousins. Her blisters were evident 3 days after birth in the generalized skin, healed without scarring, and have been continuing (Fig. 2a). She had multiple, recurrent blisters in the skin and oral mucosa, nail dystrophy, dental anomalies, alopecia, severe recurrent diarrhea, and hematuria. Endoscopic examination revealed multiple erosions on the esophagus. Congo-red staining of biopsy specimens from her intestine and kidney showed massive amyloid deposition under the epithelium (Fig. 3). Her serum level of amyloid-A fragment was $41.5 \mu \mathrm{g} / \mathrm{ml}$ (normal level $<8 \mu \mathrm{g} / \mathrm{ml}$ ). Her renal function was deteriorated. There were amyloid depositions around the skin hair follicles. Hematoxylin and eosin staining of the blistering skin lesions revealed predominant eosinophil infiltration at the perivascular zone and in the dermis just under the bulla (Fig. 2b, c). Toluidine-blue staining of the same skin section showed many mast cells beneath the blisters. These findings mimicked BP. Her serum $\operatorname{IgE}, \operatorname{IgG}, \operatorname{IgA}, \operatorname{IgM}$ levels, number of leukocytes, and
Fig. 1 The pedigree and haplotypes of family members. I At three polymorphic loci (D10S221, D10S360, and D10S541) around COL17A1 at 10q24.3. Arrow denotes the proband

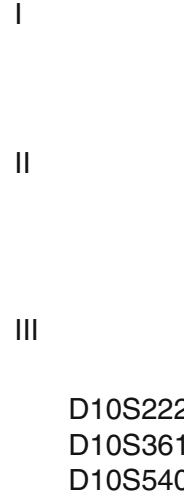

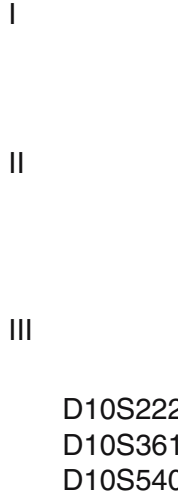

IV

D10S222

D10S361

D10S540

D10S222
D10S361
D10S540

V
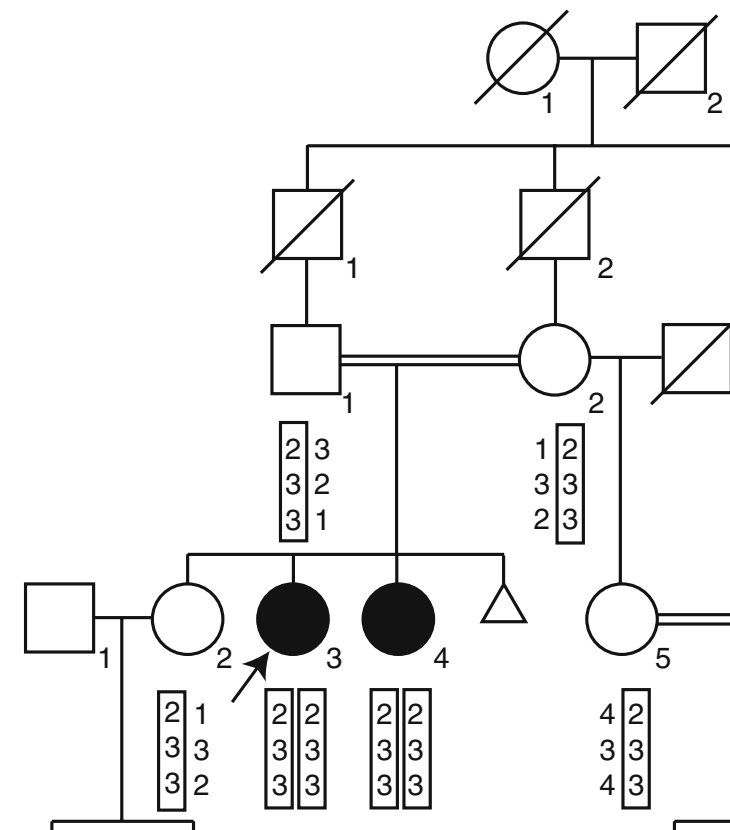
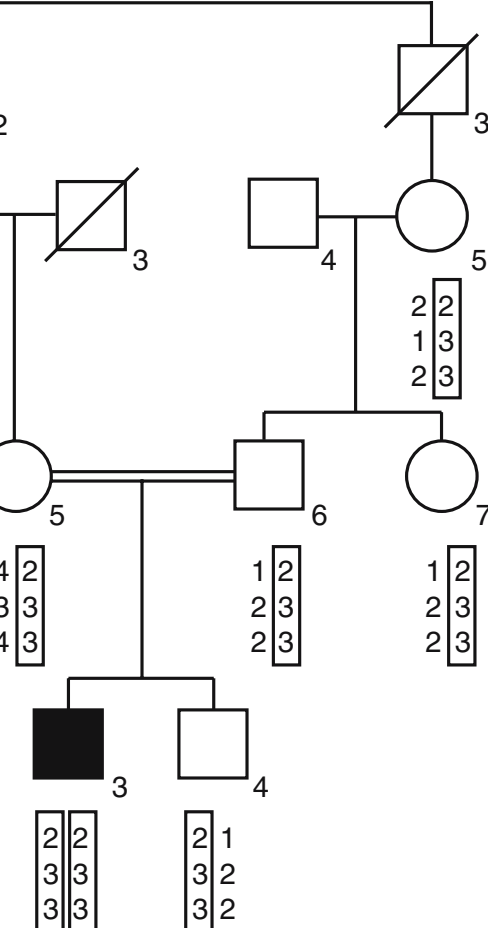
. 

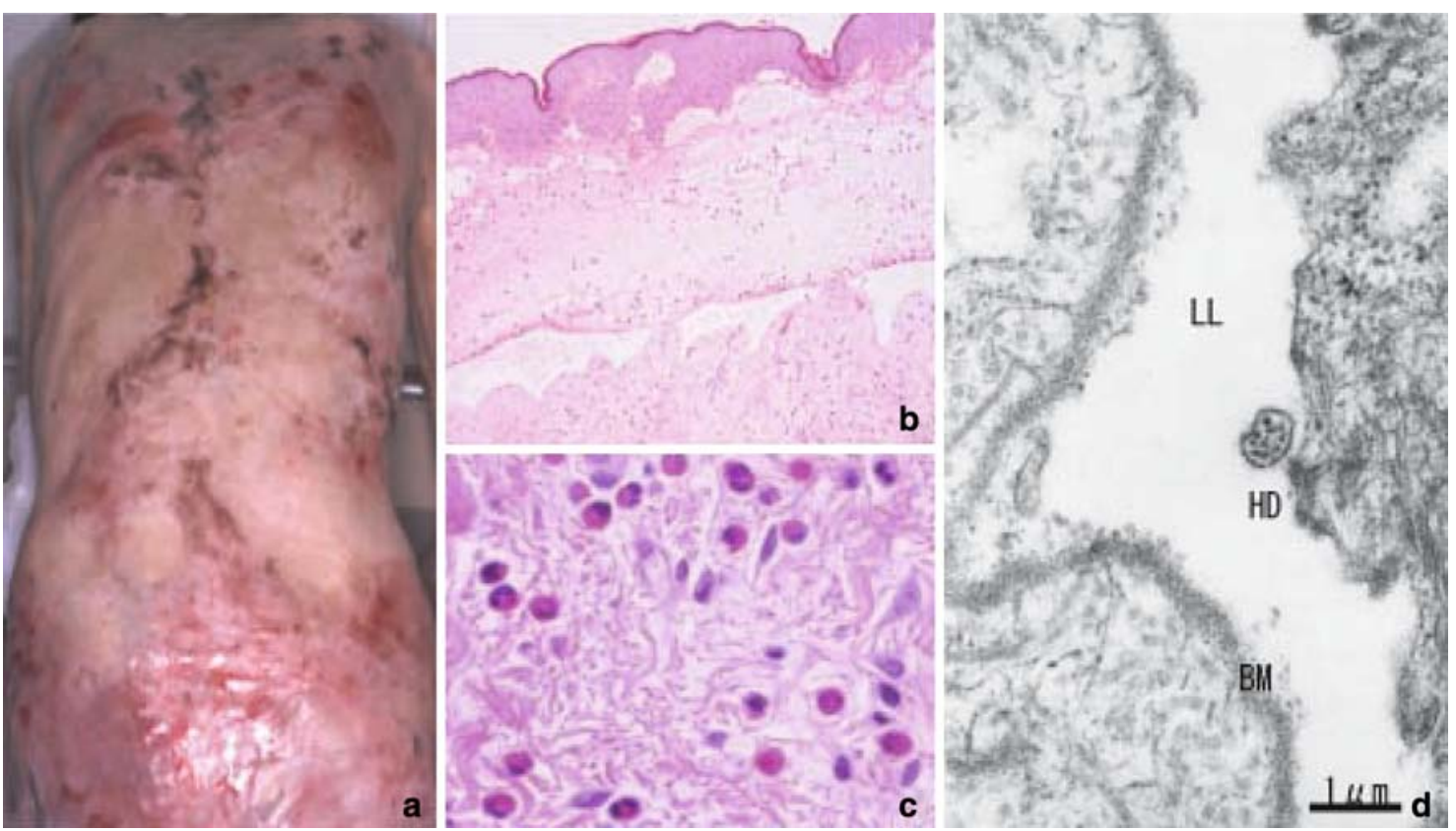

Fig. 2 Clinical and histological findings in the proband. She has blisters at different stages after trauma, healing without scars (a), eosinophil infiltration in the dermis just under the bulla $(\mathbf{b}, \mathbf{c})$, the

finding mimicking bullous pemphigoid and cleavages in the dermepidermal junction with normal lamina densa and anchoring fibrils (d). $H D$ hemidesmosomes, $L L$ lamina lucida, $B M$ basement membrane
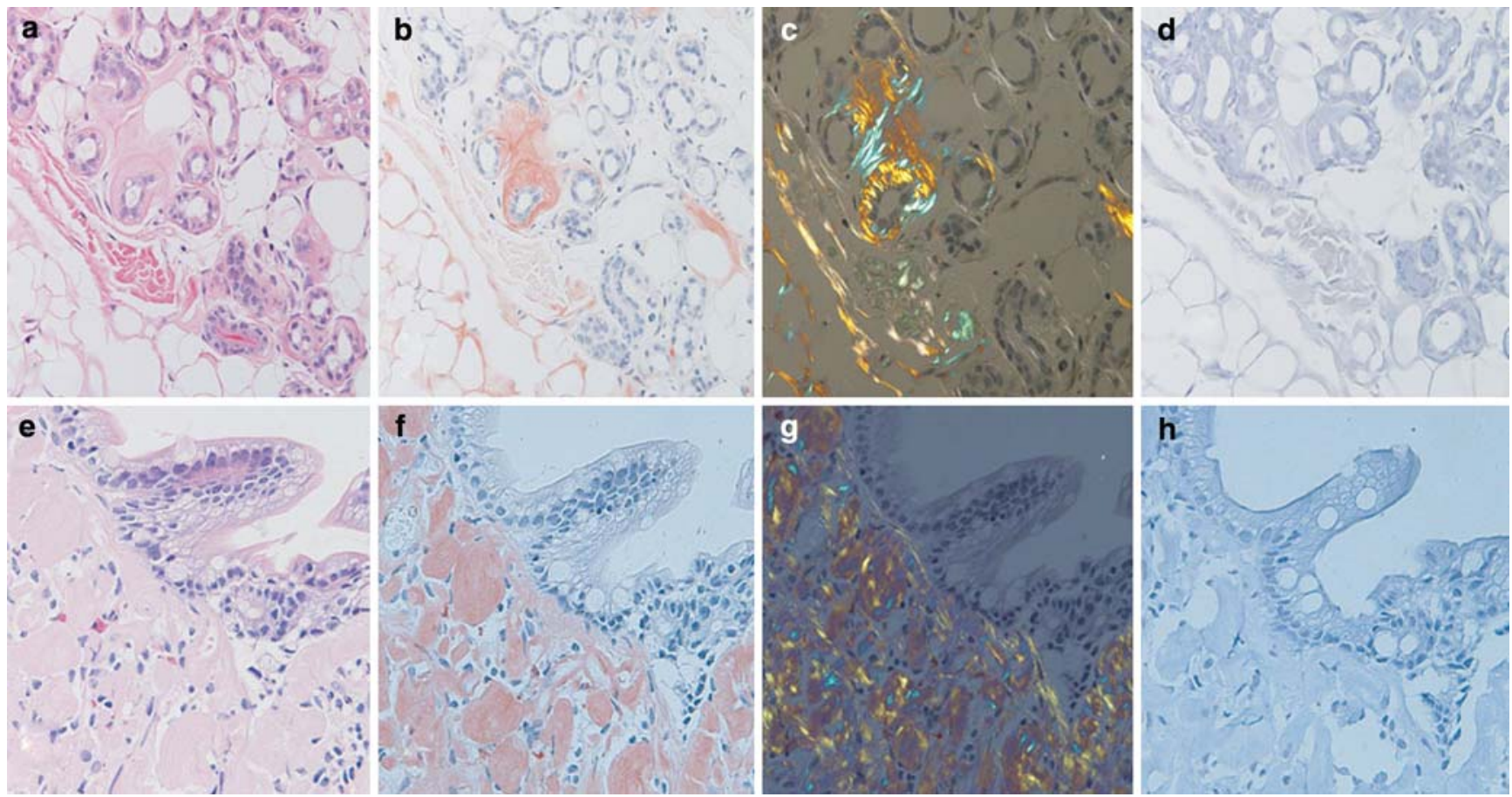

Fig. 3 Amyloid deposition in the skin (a-d) and intestine (e-h) of the proband. a and e Hematoxylin-eosin staining; $\mathbf{b}$ and $\mathbf{f}$ Congo-red staining; $\mathbf{c}$ and $\mathbf{g}$ polarized light observation of same specimens $\mathbf{b}$ and $\mathbf{f} ; \mathbf{d}$ and $\mathbf{h}$ after $\mathrm{KMnO}_{4}$ treatment of same specimens $\mathbf{b}$ and $\mathbf{f}$. Note that the amyloid was washed away by $\mathrm{KMnO}_{4}$

the eosinophil ratio in blood were normal. Direct and indirect immunofluorescent stainings never gave evidence for circulatory antibody against epidermal components and depositions of immunoglobulins/C3 on the basement membrane zone. Chemotactic factors concerning eosinophils in the blister fluid were condensed: histamine, $590 \mathrm{nmol} / 1$ (normal level $<0.5 \mathrm{nmol} /$ 1); interleukin (IL) $-5,1,610 \mathrm{pg} / \mathrm{ml}$ (normal level $<10 \mathrm{pg} /$ $\mathrm{ml}$ ); and IL-8, 2,590 pg/ml (normal level $<2.0 \mathrm{pg} / \mathrm{ml}$ ). From these findings, she was diagnosed to have GABEB 
with eosinophil and mast cell infiltration and systemic amyloidosis.

A 44-year-old younger sister (IV-4, Fig. 1) and a 39year-old nephew (V-3, Fig. 1) also had GABEB, its corresponding skin-tissue pathology, and eosinophil infiltration. Histological examinations of the two patients did not reveal any amyloid depositions. There was no family history for allergic diseases, such as atopic dermatitis, allergic rhinitis or asthma, and collagen diseases.

Electron microscopy and further immunohistological examinations

A skin sample embedded in epoxy resin was sectioned at $70 \mathrm{~nm}$, stained with uranyl acetate and lead citrate, and observed under a transmission electron microscope (JEM-1200EX, JEOL, Tokyo, Japan). Skin sections from the patients and a control individual were stained by immunohistochemical technique using various antibodies, including monoclonal antibodies against the extracellular domain (mAb-Nc16a) and the intracellular region (mAb-1A8c, a gift from Dr Katsushi Owaribe) of COL17A1.

Genetic linkage and mutation analyses

The family studied included 13 members (Fig. 1). After written informed consent was obtained under the approval of the Bioethical Board of Nagasaki University Hospital, blood samples were collected from the family members. The protocol for this study was approved by the Committee for the Ethical Issues on Human Genome and Gene Analysis in Nagasaki University. Linkage analysis was performed in the 13 family members (Fig. 1) for candidate genes or regions for EB, such as $L A M C 2, L A M B 3, L A M A 3, C O L 17 A 1$, and ITGB4, using six respective polymorphic markers around each locus. Fluorescence-labeled primers were used for genotyping of the family members studied. PCR was performed on DNA Thermal Cycler Model 9700 (Applied Biosystems, Foster City, CA, USA) in a $10 \mu \mathrm{l}$ mixture containing ExTaq DNA polymerase HS-version (TAKARA Bio Inc.). PCR products were run on an auto-sequencer Model 377 with GeneScan software (Applied Biosystems). Genotyping was carried out using Genotyper software (Applied Biosystems). To calculate LOD scores using Genehunter software (Kruglyak et al. 1996), we assumed that the disease is transmitted in an autosomal recessive mode with complete penetrance $(P=1.00)$ and set the allele frequency to be $1 / N$ when the observed number of alleles is $N$.

The genomic sequence of $C O L 17 A 1$ was retrieved from the NCBI Web site (GenBank accession no. M91669), and PCR primers for all its exons including boundary sequences were designed. PCR-based sequencing was performed using BigDye-terminator sequencing reagent version-3 (Applied Biosystems), and all sequences obtained were aligned with Autoassembler software (Applied Biosystems) and inspected for base changes.

RT-PCR analyses of mutated COL17A1 and chemotaxis-related genes and western blotting for mutated COL17A1 protein

To know whether COL17AI is processed through authentic splicing and to try to measure the amount of mutant mRNA, we performed semiquantitative RTPCR of mRNA. Total RNA was extracted using TRIZOL reagent (Invitrogen, Carlsbad, CA, USA) from the upper epidermis of a skin blister of the proband and that of a healthy person's blister, which was intentionally produced by mechanical suction. RT reaction was carried out using SuperScript ${ }^{\top \mathrm{M}}$ First-Strand Synthesis System (Invitrogen) with random hexamer oligonucleotide. PCR was carried out using the following primers $\left(5^{\prime}-3^{\prime}\right)$ : COL17A1 exon 4F/exon 7R, GACCAGCAA TGGCTATGCTA/CACTCCGTCCTCTGGTTGAAG and the relative amount of COL17A1 mRNA between the proband's and the control's keratinocytes was compared.

To know whether some specific genes are expressed together with the eosinophil infiltration, we also carried out RT-PCR analysis of the following 14 chemotaxisrelated genes: IL-3, IL-5, IL-8, IL-9, IL-10, IL-15, TNF$\alpha$, RANTES, GM-CSF, eotaxi-3, MCP-3, MCP-4, MCP-8, and NF- $\kappa \mathrm{B}$ using RNA extracted from keratinocytes.

Partial cDNA for COL17Al with an insertional mutation (209-210insCA) generated by PCR and tagged with the hemagglutinin (HA) sequence at the $3^{\prime}$-end region was cloned into pcDNA3.1(-) (Invitrogen). This construct expressing a mutant mRNA was transfected to HEK293 cells and the transformant was subjected to western blotting as previously described (Sambrook et al. 1989). Blots were treated with 2,500-fold diluted patient's serum as a primary antibody, followed by treatment of HRP-conjugated secondary antibodies against human $\operatorname{IgG}, \operatorname{IgM}, \operatorname{IgA}$, or $\operatorname{IgE}$ and finally visualized using chemiluminescence (Amersham Biosciences, Buckinghamshire, UK).

\section{Results}

The linkage analysis and subsequent haplotyping revealed that GABEB in the family was linked to three polymorphic marker loci (D10S221, D10S360, and D10S541) around COL17A1 at 10q24.3 with the maximum LOD score of 3.08. The results suggested COL17A1 as the causative gene for GABEB in that family. Sequencing analysis of the patients identified a homozygous 2-bp insertional mutation, 209-210insCA, 


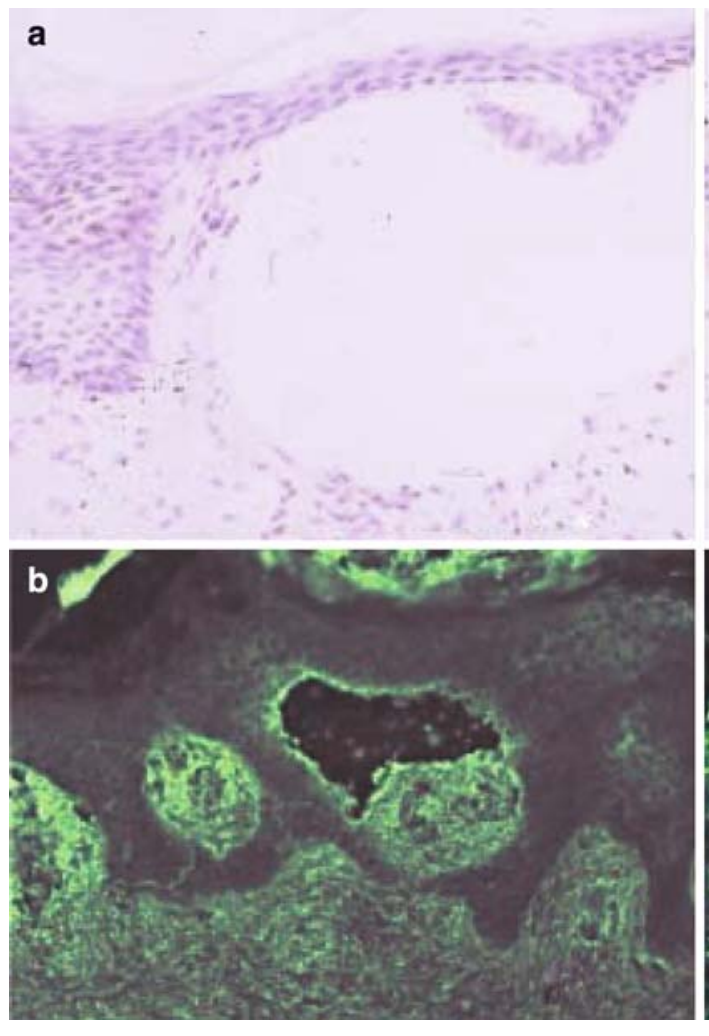

Fig. 4 Immunohistological findings in the proband's skin $(\mathbf{a}, \mathbf{b})$ and in the normal skin from a control individual (c, d). a and c Immunofluorescence staining using an antibody, $1 \mathrm{~A} 8 \mathrm{c}$, against the intracellular region; $\mathbf{b}$ and $\mathbf{d}$ Immunofluorescence staining using an

in the exon 5 of COL17Al (Fig. 5a). The insertion is predicted to create a premature termination codon before the transmembrane domains. The semiquantitative RTPCR products from a mutated and a wild-type COL17 A1 mRNA from the proband's and normal skin, respectively, revealed a single band on agarose gel electrophoresis and almost the same amount (Fig. 6). Sequencing of the RT-PCR product of the proband ruled out the possibility of exon skipping. The RT-PCR analysis of the 14 eosinophil chemotaxis-related genes did not detect any differential expression between the skin specimens from the proband and the control (data not shown).

Electron microscopy of the skin specimen from the proband revealed cleavages in the derm-epidermal junction (Fig. 2d) with a normal appearance of both the lamina densa and the anchoring fibrils. Immuno-

Fig. 5 Partial electropherogram of $C O L 17 A 1$ (a) and schematic representation of BP180 (b). A 2-bp insertion, 209-210insCA, in exon 5 of $C O L 17 A 1$ was identified in the proband (upper row in a) and the wild-type sequence in a normal control individual (lower row in a). The mutation of the proband is located at the most 5 -position among previously reported mutation sites (arrow). $\mathrm{N}$-terminus is located within the cytoplasm of the keratinocyte, extracellular domain traverses the lamina lucida, and C-terminus is situated in the lamina densa. The $1 A 8 c$ and $N c 16 a$ are domain names of the cytoplasmic region and the extramembrane region in the BP180, respectively
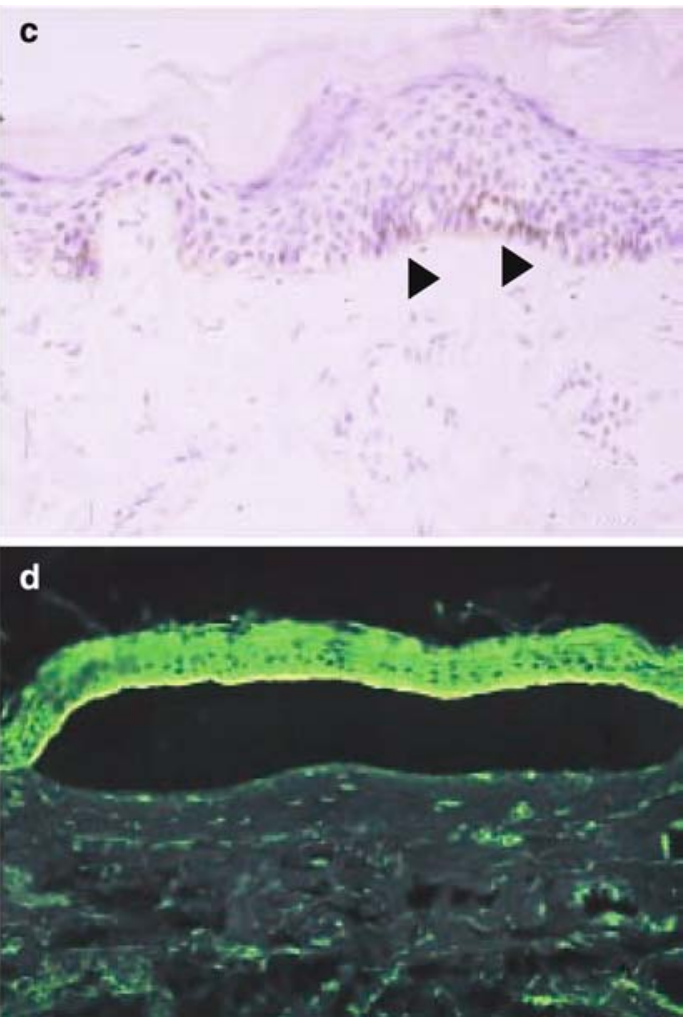

antibody, Nc16a, against the extracellular domain of BP180. No fluorescent signal is obvious on the proband's skin, compared to the normal skin. Triangles depict brownish deposition of antibodies as positive staining

histochemical analysis of the specimen using antibodies against the intracellular region of COL17A1 showed no fluorescent signal along the basement membrane

a
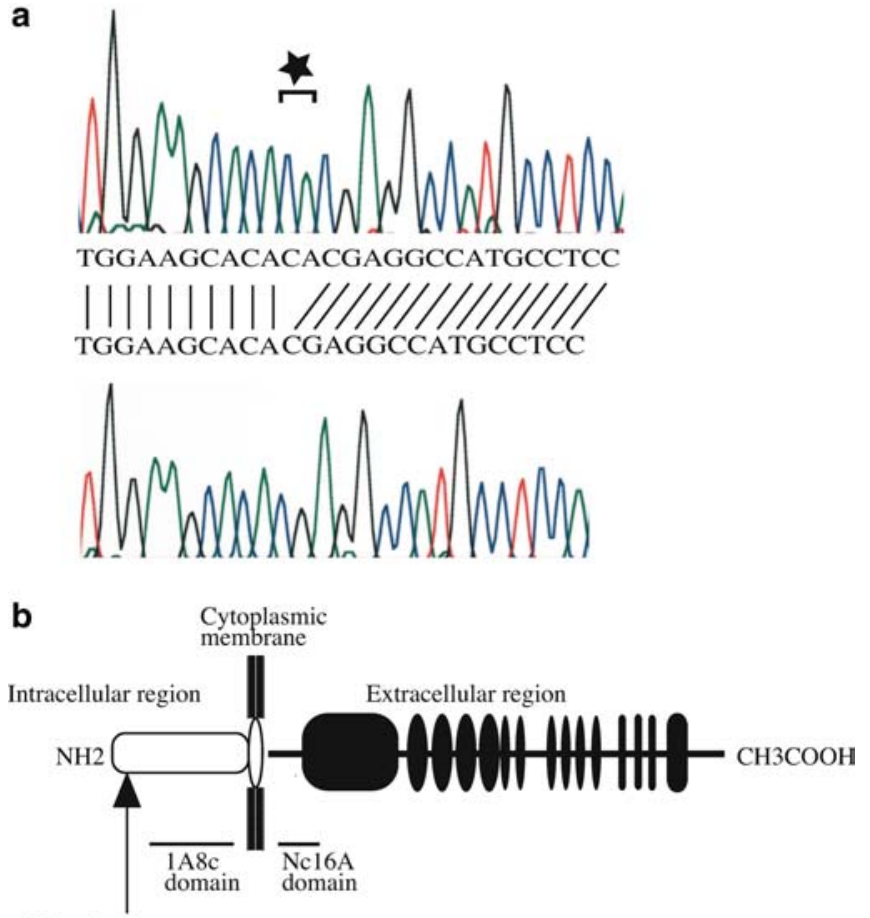

Mutation site 
(Fig. 4a). Immunofluorescent signal was also lacking when using an antibody against an extracellular domain of COL17A1 (Fig. 4b). Analysis using other antibodies against keratine-5, keratine-14, alpha-4/beta-6 integrin, BP230, collagen IV, and plectin showed signals on the roof (upper epidermis) of the blisters, while the laminin $\mathrm{V}$ and collagen VII signals appeared at the bottom (basement membrane) of the blisters. No alteration of these signals was seen in the proband's skin, compared to the normal one (data not shown). Western blotting of frameshift COL17A1 protein, forced to be expressed in mammalian cells, revealed no evidence of the existence of autoantibodies against the mutant protein in the patients' serum (data not shown).

\section{Discussion}

The three patients in the family we described were clinically diagnosed to have GABEB. Their clinical history (the onset of the disease 3 days after birth in the proband), inheritance mode (two pairs of consanguineous parents), characteristic skin lesions (cleavage in the lamina lucida and normal lamina densa and anchoring fibrils), and other associated manifestations (nail dystrophy, dental anomalies, and alopecia) all led to the diagnosis (Hinter and Wolf 1982; Fine et al. 2000). Genetic analysis of the patients disclosed a novel, homozygous insertional mutation, 209-210insCA, in exon 5 of $C O L 17 A 1$. The insertion is located at the most 5 '-region among previously reported mutation sites (Bauer and Lanschuetzer 2003). The mutation is expected to create a premature termination codon in exon 9. Consequently, of the 173 amino acids translated, 71 at the $\mathrm{N}$-terminus reflected the $5^{\prime}$ wild-type sequence and the remaining 102 were derived from frameshift sequences (Fig. 5b). It is thus most likely that the mutation results in the fragility of attachment between the epidermis and basement membranes, leading to GABEB. Huber et al. (2002) stated that COL17A1 responsible for nonsense mutation-mediated mRNA decay was accelerated in keratinocytes to eliminate the mutant transcripts through the highly conserved mechanism. Nevertheless, the altered COL17A1 protein that retains 71 wild-type amino acids within the intracellular region, albeit nonfunctional, is probably produced in our patients' keratinocytes, because the mutated mRNA was confirmed to be transcribed through authentic splicing and its amount was almost the same as wildtype mRNA (Fig. 6).

Histological observations in our patients, i.e., massive eosinophil infiltration without neutrophils, which occurred $6 \mathrm{~h}$ after intentional introduction of bulla, in the dermis just under the bulla and elevated eosinophil-related cytokines in blister fluid, are unusual in GABEB but resemble BP (Van den Bergh and Giudice 2003). Although BP and GABEB are distinct diseases, i.e., the former is an acquired autoimmune disease and the latter a heritable disorder, a common immunological

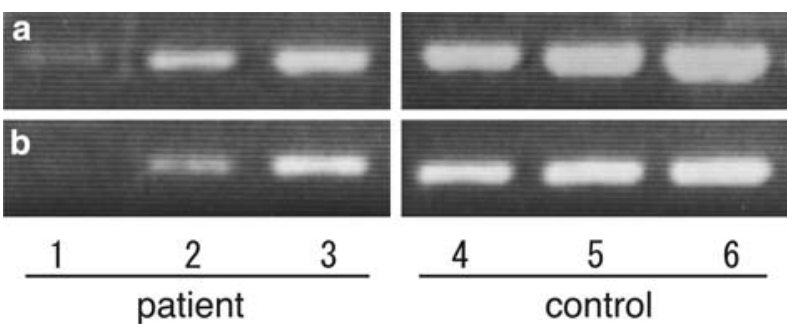

Fig. 6 Semiquantitative RT-PCR for $C O L 17 A 1$. The cDNA of COL17A1 (panel $a$ ) and of GAPDH (panel $b$ ) were amplified by RT-PCR using epidermal tissue RNA. Random primed RT products were subjected to various cycles of PCR amplification. Lanes 1 and 4, 36 cycles; lanes 2 and 5,38 cycles; and lanes 3 and 6, 40 cycles. Relative intensity ratio of $C O L 17 A 1$ to $G A P D H$ in the proband (lanes 1-3) is not decreased compared to the control sample (lanes 4-6)

mechanism would exist for the derm-epidermal detachment between BP and GABEB in our patients. Eosinophil infiltration is one of the characteristic early events in BP (Sams and Gammon 1982) and degranulation of eosinophils that invade into the blister's fluid and release their related cytokines may be a part of immune reactions involved in the derm-epidermal detachment in BP (Engineer et al. 2001; Bowszyc-Dmochowska and Dmochowski 2002; Van den Bergh and Giudice 2003). It is also likely that anti-COL17A1 antibodies, such as those against the COL17A1 extracellular domain (Nc16a) (Zillikens et al. 1997), play a main role in the pathogenesis of BP because the disease is associated with tissue-bound and circulating IgG-type autoantibodies against the basal membrane zone of the stratified epithelium (Perriard et al. 1999; Liu and Diaz 2001). Recent studies showed that some BP patients have immunereactivity against the intracellular domain of COL17A1 (1A8c) in an early stage of the disease and suggested an important role of intracellular epitopes in the initiation and development of BP (DiZenzo et al. 2004). These findings led us to assume that our patients' truncated COL17A1 retaining a portion of the intracellular domain would be more or less expressed and consequently a certain autoantibody would invoke an immune reaction to involve eosinophils. However, by the western blot analysis, such a mutant protein was not detected in the conditional medium, but identified only in the HEK293 cells when using $1 \times$ sample lysis buffer (containing 3\% SDS) directly onto the cell in the culture dish. Therefore, we concluded that a truncated COL17A1 was prone to be degraded when cells were replaced in nonphysiological conditions, retained in the cell, and were not secreted (data not shown). No autoantibodies were detected in the serum of our patients or deposited at their basement membrane zone. Furthermore, no antibody-mediated immune response on the basement membrane was seen by the immunohistochemical analysis. It is plausible that the misfolded mutant protein has led to cell death and abnormally triggered an immune response. 
Eosinophil infiltration has also been seen in infants with some types of EB, such as epidermolysis bullosa simplex, junctional epidermolysis bullosa, dominant dystrophic epidermolysis bullosa, but mutations in patients with these disorders were not confirmed (Roth et al. 1990; Anton-Lamprecht and Schnyder 1982; SanzSantamaria and McNutt 1966; Grunwald et al. 1999). Eosinophil infiltration seen in the neonates with EB was assumed to be a phenomenon of their immaturity and/or insufficiency of the immune response system (Roth et al. 1990; Grunwald et al. 1999). In our patients, eosinophil infiltration was also observed in the ileum. Various degrees of amyloid depositions were observed in many tissues of the proband, such as the skin, ileum, and kidney. Amyloid deposition in the proband may be a secondary phenomenon because this was not seen in the other two patients of the family. Although the mechanism for the production of amyloid is unknown, amyloid deposition is likely a result of an abnormal immune response against continuous inflammation.

In conclusion, since there is no direct evidence for a local antigen-antibody reaction in our GABEB patients, their pathological mechanism of eosinophil infiltration remains unknown. Mechanical stress and/or exposures to external atmosphere might invoke a unique reaction in the skin through unknown mechanisms.

Acknowledgements We are indebted to the family members for their participation in this research. We especially thank Professor Katsushi Owaribe at Nagoya University, Japan, and Professor Takashi Hashimoto at Kurume University School of Medicine, Japan, for providing antibodies. N.N. was supported in part by a Grant-in-Aid for Scientific Research (Category S, No. 13854024) from the Ministry of Education, Culture, Sports, Science and Technology (MEXT) of Japan and by CREST from the Japan Science and Technology Agency (JST). K.Y. was supported by Grant-in-Aid for Scientific Research for Priority Areas (No. 15590291) from MEXT of Japan. We also thank Ms. N. Yanai, Y. Noguchi, and K. Miyazaki for their technical assistance.

\section{References}

Anton-Lamprecht I, Schnyder UW (1982) Epidermolysis bullosa herpetiforms Dowling-Meara: report of a case and pathomorphogenesis. Dermatologica 164:221-235

Bauer JW, Lanschuetzer C (2003) Type XVII collagen gene mutations in junctional epidermolysis bullosa and prospects for gene therapy. Clin Exp Dermatol 28:53-60

Bowszyc-Dmochowska M, Dmochowski M (2002) Immediate hypersensitivity phenomena in bullous pemphigoid: critical concepts. J Med 33:189-198

DiZenzo G, Grosso F, Terracina M, Mariotti F, DePita O, Owaribe K, Mastrogiacomo A, Sera F, Borradori L, Zambruno G (2004) Characterization of the anti-BP180 autoantibody reactivity profile and epitope mapping in bullous pemphigoid patients. J Invest Dermatol 122:103-110
Engineer L, Bhol K, Kumari S, Ahmed R (2001) Bullous pemphigoid: interaction of interleukin 5, anti-basement membrane zone antibodies and eosinophils: a preliminary observation. Cytokine 13:32-38

Fine JD, Eady RAJ, Bauer EA, Briggaman RA, Bruckner-Tuderman L, Christiano A, Heagerty A, Hinter H, Jonkman MF, McGrath J, McGuire J, Moshell A (2000) Revised classification system for inherited epidermolysis bullosa: report of the second international consensus meeting on diagnosis and classification of epidermolysis bullosa. J Am Acad Dermatol 42:1051-1066

Fine JD, Johnson LB, Weiner M, Stein A, Cash S, DeLeoz J, Devries DT, Suchindran C (2004) Inherited epidermolysis bullosa and the risk of death from renal disease: experience of the National Epidermolysis Bullosa Registry. Am J Kidney Dis 44:651-660

Grunwald MH, Amichai B, Avinoach I, Kedar T, Bergman R (1999) Dystrophic epidemolysis bullosa associated with eosinophilic infiltrate and elevated serum IgE. Pediatr Dermatol 16:16-18

Hinter H, Wolf K (1982) Generalized atrophic benign epidermolysis bullosa. Arch Dermatol 118:375-384

Huber A, Yee C, Darling TN, Yancey KB (2002) Comprehensive analysis of gene expression profiles in keratinocytes from patients with generalized atrophic benign epidermolysis bullosa. Exp Dermatol 11:75-81

Jean-Baptiste S, O'Toole EA, Chen M, Guitart J, Paller A, Chan LS (2002) Expression of eotaxin, an eosinophil-selective chemokine, parallels eosinophil accumulation in the vesiculobullous stage of incontinentia pigmenti. Clin Exp Immunol 127:470-478

Kruglyak L, Daly ML, Reeve-Daly MP, Lander ES (1996) Parametric and nonparametric linkage analysis: a unified multipoint approach. Am J Hum Genet 58:1347-1363

Liu Z, Diaz LA (2001) Bullous pemphigoid: end of the century over view. J Dermatol 28:647-650

Perriard J, Jaunin F, Favre B, Budinger L, Hertl M, Saurat JH, Borradori L (1999) IgG autoantibodies from bullous pemphigoid (BP) patients bind antigenic sites on both the extracellular and the intracellular domains of the BP antigen. 180. J Invest Dermatol 112:141-147

Roth RR, Smith KJ, James WD (1990) Eosinophilic infiltrates in epidermolysis bullosa. Arch Dermatol 126:1191-1194

Sambrook J, Fritsch EF, Maniatis T (1989) Molecular cloning: a laboratory manual, 2nd edn. Cold Spring Harbor Laboratory Press, NY, USA

Sams WM, Gammon WR (1982) Mechanism of lesion production in pemphigus and pemphigoid. J Am Acad Dermatol 6:431-449

Sanz-Santamaria MC, McNutt SN (1966) Epidermolysis bullosa with eosinophils; the role of the mast cells. Acta Dermosifiliogr $87: 19-25$

Schumann H, Hammami-Hauasli N, Pulkkinen L, Mauviel A, Kuster W, Luthi U, Owaribe K, Uitto J, Bruckner-Tuderman L (1997) Three novel homozygous point mutations and a new polymorphism in the COL17A 1 gene: relation to biological and clinical phenotypes of junctional epidermolysis bullosa. Am J Hum Genet 60:1344-1353

Van den Bergh F, Giudice GJ (2003) BP180 (type XVII collagen) and its role in cutaneous biology and disease. Adv Dermatol 19:37-71

Zillikens D, Rose PA, Balding SD, Liu Z, Olague-Marchan M, Diaz LA, Giudice GJ (1997) Tight clustering of extracellular BP180 epitopes recognized by bullous pemphigoid autoantibodies. J Invest Dermatol 109:573-579 